\title{
Royal Jelly ameliorates 6-mercaptopurine induced spermatogenesis impairment and testicular apoptosis by regulating PI3K/AKT pathway in male rats
}

khalid Hashem ( $\nabla$ khalidshaabanhashem@yahoo.com )

Beni Suef University https://orcid.org/0000-0001-5303-4739

Ahmed Z. Abdelazem

Beni Suef University

Naglaa W. Abdelbaky

Beni Suef University

Research

Keywords: 6MP, male infertility, testicular dysfunction, FSH, LH and Androgen receptor

Posted Date: January 8th, 2020

DOl: https://doi.org/10.21203/rs.2.20288/v1

License: (c) (1) This work is licensed under a Creative Commons Attribution 4.0 International License.

Read Full License 


\section{Abstract}

Testicular apoptosis is an obvious adverse effect of many chemotherapeutic agents.one of these chemotherapeutic drugs is 6-mercaptopurine (6MP) which has a powerful anticancer effect. On the contrary, it has an adverse effect on the male reproductive system. This study aimed to evaluate the prospective ameliorative effects of Royal Jelly (RJ) on 6MP induced testicular apoptosis and investigate the mechanistic pathway of protection. For this aim, forty male adult albino rats were divided into four equal groups ( $n=10)$ : control rats, RJ group ( $200 \mathrm{mg} / \mathrm{kg}$.b.wt. of RJ for 30 day P.o.), $6 \mathrm{MP}$ group (5 $\mathrm{mg} / \mathrm{kg}$.b.wt of 6MP for 20 day P.o.), and RJ+6MP group pretreated with RJ (200 mg/kg.b.wt. for 10 day P.o.), and continued with $6 \mathrm{MP}$ (5 mg/kg.b.wt, P.o) for 20 day. After 30 days blood samples, epididymis and testis were collected to investigate sex hormones, sperm parameters, histological and molecular changes of testicular tissues, that include anti-oxidants activity, caspase-3, TNF-a, gene expression of Androgen receptors (AR) and P53 also protein concentration of PI3K, AKT, Nrf2 and H01 were estimated. The results of our study revealed that Pretreatment of Royal Jelly (RJ) abrogated 6MP induced spermatogenesis impairment by ameliorating sperm count, motility and morphology, regulating AR mRNA expression and sex hormones levels. RJ ameliorated testicular damage of 6MP exposed rats through restoring testicular antioxidant/oxidative redox, inhibiting caspase-3 activity and P53 mRNA expression as well as regulation of $\mathrm{PI} 3 \mathrm{~K}, \mathrm{AKT}, \mathrm{Nrf} 2$ and $\mathrm{HO} 1$ protein levels. Our data concluded that RJ protected testicular tissue from 6MP induced apoptosis by regulation PI3K/AKT pathway.

\section{Introduction}

6-Mercaptopurine (6MP) was the first purine analog to prove beneficial for neoplastic diseases treatment [1]. It is used in cancer therapy to maintain the remission of acute lymphoblastic leukemia [2]. 6MP has a potent powerful immunomodulatory effect in treatment of inflammatory bowel disease, systemic lupus erythromatosus, organ transplant rejection and rheumatoid arthritis [3].

6MP mechanism of action is incorporation of 6-thiodeoxygoansine nucleotides into DNA during DNA synthesis process. The antagonistic effect of $6 \mathrm{MP}$ on purine bases could results in alteration in DNAprotein interaction or DNA mismatch repair, therefore induces cytotoxicity [1].

Efficacy of $6 \mathrm{MP}$ is associated with various adverse effects on body organs induced toxicity as hepatotoxicity [4], bone marrow suppression and infertility [5]. 6MP induced male reproductive organ toxicity include testicular atrophy, Leydig cell failure and spermatogenesis impairment [6] .

Recent trends in treating diseases promote uses of natural products to protect the body against the adverse effects of some chemotherapeutic drugs [7]. Royal Jelly (RJ) is one of the natural products which considered an effective adjuvant to chemotherapies. RJ is secreted by hypo-pharyngeal glands of worker honeybee and consider a principal food of queen honeybee, RJ components are important for cell growth and tissue repair, it is consists of water (50-60\%), proteins (18\%), carbohydrates (15\%), lipids (3-6\%), mineral salts (1.5\%), and vitamins [8]. Previous studies reports that RJ act as anti-inflammatory, 
antioxidant and lower serum cholesterol and glucose levels when used as adjuvant to certain drugs [9, 10]. RJ enhanced spermatogenesis and ameliorated the adverse effects some drugs on the reproductive organs $[11,12]$. The underlying protective mechanism of RJ against 6MP induced testicular apoptosis and oxidative stress is not clearly studied. Therefore, our study focused on the impact of RJ to prevent reproductive complications in male albino rats when used as adjuvant to 6MP, and following the mechanistic pathway of protection through measuring biochemical, molecular, and histological changes occurred in the testis.

\section{Material And Methods \\ 2.1. Chemicals}

Royal jelly was obtained from GlaxoSmithKline Company, Egypt. RJ capsule (1 gm) was opened and dissolved in physiological saline ( $0.9 \% \mathrm{Nacl})$. 6-mercaptopurine ( $5 \mathrm{gm}$ package) were purchased from Sigma-Aldrich Company, USA, 6MP powder was grinding then suspended in physiological saline $(0.9 \%$ $\mathrm{Nacl}$ ). MDA and antioxidant parameters were estimated using commercial kits (Bio-diagnostic for Research Kits, Egypt). Molecular kits were obtained from QIAGEN Company, USA. Other kits and tools were purchased from high quality sources

\subsection{Experimental animals}

Forty adult male albino rats, 8 weeks old, weighting an average $180-200 \mathrm{~g}$ were transferred to the animal house of biochemistry laboratory two weeks prior to the experiment initiation for acclimatization. The rats were housed under standard laboratory conditions maintained at 12-hour light / day cycle and ambient temperature of $25 \pm 2{ }^{\circ} \mathrm{C}$. All experiments were conducted in accordance with the criteria of the investigations and Ethics Committee of the Community Laws governing the use of experimental animals of Beni-suef University.

\subsection{Experimental design:}

After the adaptation period, rats were randomly assigned to 4 groups ( $\mathrm{n}=10$ rats): group I (control group); rats received saline orally by gastric gavage tube for 30 days, Group II (RJ group); rats received RJ (200 mg/kg B.W.) for 30 days [13], Group III (6MP group); rats received saline (0.9\% Nacl) for 10 successive days followed by $6 \mathrm{MP}(5 \mathrm{mg} / \mathrm{kg} \mathrm{B}$. W.) daily for 20 day. Group IV (RJ + 6MP): Rats received RJ (200 mg/kg B.W.) daily for 30 days, at the 10th day rats administered $6 \mathrm{MP}(5 \mathrm{mg} / \mathrm{kg} \mathrm{B}$. W.) for 20 days

\subsection{Blood sampling and tissue collection:}

After 24 hours from the last administered dose, under ether anesthesia, blood was collected from the medial canthus blood capillaries of the eye by capillary tube [14] in labeled dry centrifuge tubes which were leaved for coagulation then were centrifuged at $1000 \mathrm{~g}$ for 20 minutes. Clear sera were separated in labeled Eppendorf tubes and preserved in deep freezer (-80®C) until analysis for detection of sex hormones. 
At the time of sacrifice, the animals were euthanized by cervical dislocation (dislocation of spinal cord column from brain by applying pressure to neck). Testis were dissected out and weighed. The right testis was immediately fixed in $10 \%$ neutral formalin for histomorphological study and the left testis was washed in physiological saline then silicate into two parts, the first part was homogenized by using homogenizer (Ortoalresa, Spain) for biochemical analysis. The second part was used for molecular investigation (real time PCR and western blotting techniques).

\subsection{Weighting testes, epididymis and Semen evaluation}

After scarification of rats, the testes were removed along with its epididymis and weighted separately. Also, The Testes and epididymis were separately immersed in $5 \mathrm{ml}$ normal saline in a measuring cylinder and recording its volumes. The cauda epididymis were isolated and immersed immediately in physiological saline $(0.9 \% \mathrm{NaCl})$, then incubated to allow spermatozoa separation from epididymis tubules, supernatant was added by percentage (1:100) to solution contains $1 \mathrm{ml}$ formalin (35\%), $25 \mathrm{mg}$ of Eosin, $5 \mathrm{gm}$ of $\mathrm{NaHCO} 3$ in $100 \mathrm{ml}$ water [15]. For sperm counting, $10 \mu \mathrm{l}$ of the prepared mixture was transferred to the counting champers of homocytometer under light microscope (Sanli, China Mainland), stand for 5 minutes and count sperms at $40 \mathrm{X}$. For sperm viability determinations, equal volumes of sperm solution and Eosin-Nigrosin stain were mixed, spread thin film on clean slide and examined under light microscope to count dead (pink) sperms and live (unstained) sperms, then calculate the ratio of viable sperms in the sample [16]. Determination of sperms motility was done by addition of semen solution to Tris-buffer, and count motile sperms at $40 \mathrm{X}$ in comparison with total sperms count. Abnormal forms were examined also at $40 \mathrm{X}$, and recorded to calculate its percentage [17].

\subsection{Histopathological examination}

Testis was fixed in $10 \%$ neutral formalin for 48 hours. And then processed according to Bancroft and Gamble (2008) [18], then washed in running water, graduated ethanol $(50 \%, 70 \%, 95 \%$ then $100 \%)$ respectively, $2 \mathrm{~h}$ for each, cleared in xylene and embedded in paraffin wax at $70{ }^{\circ} \mathrm{C}$, blocking in paraffin and then undermined in microtome. Tissues sections were mounted on $4 \mu-6 \mu$ on clean glass slides and stained by Hematoxylin and Eosin (H\&E). Microscopically examined and photographed by using light microscope (Sanli, China Mainland). The histological sections were examined for the presence of congestion, tubular degeneration, cellular vacuolation and Pyknosis of cell lining seminephrous tubules. The histological changes were scored from 0 to 3 according to the histological findings, where 0 represents no pathological findings, and 1, 2 and 3 represent pathological findings in $<33 \%, 33-66 \%$ and $>66 \%$ of the testes, respectively. The scores for each parameter were calculated and the total scores were obtained and presented as means \pm SEM.

\subsection{Tissue preparation of the homogenate}

$0.5 \mathrm{~g}$ of testis was homogenized in $5 \mathrm{ml}$ physiological saline and centrifuged at $20,000 \times \mathrm{g}$ for $30 \mathrm{~min}$ at $4{ }^{\circ} \mathrm{C}$. The supernatants were collected and stored at $-80^{\circ} \mathrm{C}$ for the assessment of reduced glutathione(GSH), glutathione reductase (GR), catalase, superoxide dismutase(SOD), lipid peroxidation (MDA), caspase-3, TNF-a 


\subsection{Sex hormones assay}

Enzyme Linked Immunosorbent Assay (ELISA) kit ( MyBioSource Company, USA ) was used for estimation of serum levels of testosterone, FSH ( follicular stimulating hormone) and LH (leutinizing Hormone ) following the instructions of kits protocol, the principle is based on antigen antibody reaction.

\subsection{Oxidant/antioxidant assay}

The testicular content of lipid peroxidation represented in Malondialdehyde (MDA) was measured according to protocol described by Ohkawa (1979), assay is based on reaction of MDA with thiobarbituric acid to form reactive colored product, absorbance of colored product can be measured at $534 \mathrm{~nm}$, concentration of MDA calculated from standard curve using standard concentrations [19]. Catalase activity in testis sample was estimated using hydrogen peroxide substrate according to instructions described by Aebi, H. (1984) [20]. Super oxide dismutase was estimated according to the method described by Koracevic et al.(2001) [21]. glutathione reduced (GSH) was assayed colorimetrically, follow the method of Beutler E. (1963), the method based on the reduction of 2-nitrobenzoic acid through reaction with glutathione reduced to produce colored compound, reduced chromogen absorbance can be measured at $405 \mathrm{~nm}$, and its concentration is directly proportional to the concentration of GSH [22]. Glutathion reductase (GH) assay was performed according to the method of Brigelius, et al [23]

\subsection{ELISA assay for measuring Caspase-3 and TNF-a}

Caspase-3: was assayed by rat caspase-3 ELISA kit according to instructions of manufacturer MBS1602954 MyBioSource Company, USA

Tumor Necrosis Factor- alpha (TNF-a) level: was estimated as a pro-inflammatory marker using rat TNF-a ELISA kits according to manufacturer's instructions of MyBioSource Company, USA (MBS 490379) [24]

\subsection{Western blotting assay for assessing PI3K, AKT, Nrf2 and $\mathrm{H} 01$}

Testicular samples were homogenized using RIPA lysis buffer (buffer enables protein extraction from cytoplasmic membrane and nuclear proteins), the lysate was kept on ice for 30 minutes on shaker, then centrifuged at $\sim 16,000 \mathrm{xg}$ for 30 minutes at $4{ }^{\circ} \mathrm{C}$, cell debris was removed and supernatant was transferred to a new tube for further protein concentration determination analysis.

Quantitative estimation of protein was assayed according to instructions of Bradford Protein Assay Kit (Markham Ontario L3R 8T4 Canada). Extracted protein in each sample was added to equal volume of loading buffer(4\% SDS, 20\% glycerol, 10\% 2-mercaptoethanol, 0.125MTris $\mathrm{HCl}, 0.004 \%$ bromophenol blue), the mix was boiled at $95{ }^{\circ} \mathrm{C}$ for 5 minutes. Protein in loading buffer were electrophoresed using $10 \%$ SDS PAGE then electro-transferred to polyvinylidene diflouride(PVDF) membranes. The membrane was blocked in tris-buffered saline with Tween 20 buffer and 3\% bovine serum albumin (BSA) for $1 \mathrm{hr}$. The membrane was incubated overnight with anti PI3K, AKT, Nrf2 and HO1 antibodies (Thermofisher. USA) 
separately, Beta actin was used as control. After washing 4 times for 5 min each in DPBS (tween-20), the blot was incubated with the corresponding Goat anti-rabbit IgG for $1 \mathrm{~h}$, washed and then developed, finally protein band images were recorded in BioMax film, and quantified using densitometry and Molecular Analyst Software (Bio-Rad, Richmond, CA, USA).

\subsection{DNA fragmentation assay}

DNA was extracted from testicular tissue by using chemical method of DNA purification [25], DNA fragmentation percentage was estimated by addition of tetron-X-buffer with vortex for fragmented DNA releasing, then addition of tetrachloroacetic acid(5\%) and heated at $90^{\circ} \mathrm{C}$ for 15 minuts. Samples were measured calorimetrically with diphenylamine stain at $600 \mathrm{~nm}$. Gel electrophoresis analysis for the DNA was done according to the method described by $\mathrm{K}$ Weber, M Osborn [26], finally gels were illuminated with ultraviolet light at $300 \mathrm{~nm}$ and photographic record was made to show the fragmented DNA in comparison with DNA ladder .

\subsection{Quantitative Real time polymerase chain reaction (RT- qPCR): P53 mRNA and androgen receptors (AR) mRNA expression}

Gene expression was estimated through three steps. i) RNA extraction, ii) complementary DNA (cDNA) synthesis, iii) Real time qPCR assay. (i)RNA in testicular tissues (homogenate) was isolated using Qiagen tissue extraction kit (Qiagen, USA) according to instructions of manufacture, lysis buffer TLT was added to $30 \mathrm{mg}$ of testis tissues, the tissue was lysed and homogenized using homogenizer for 40 seconds, the lyset was centrifuged for 3 min and the supernatant was transferred into microcenterifuge tube, $350 \mu$ of 705 ethanol was added to the cleared lysat on RNeasy spin column high to washed three times using wash buffer of the kits, RNeasy spin column was placed in a new $1.5 \mathrm{ml}$ collection tube. $50 \mu \mathrm{l}$ RNase-free water was added directly to the spin column membrane, and centrifuged for $1 \mathrm{~min}$ at $\geq 8000 \mathrm{rpm}$ to elute the RNA. The concentration of RNA was obtained using nano drop spectrophotometry. (ii)The produced total RNA $(0.5-2 \mu \mathrm{g})$ was used as a template for CDNA synthesis with random primers using high capacity cDNA reverse transcription kit( Fermentas, USA). cDNA master mix was prepared according to kit's instructions and was added to RNA-Primers mixture to produce cDNA. (iii)Real-time qPCR reaction were performed using (Applied Biosystems, USA) with software version 3.1 (StepOne ${ }^{\mathrm{TM}}$, USA)., gene expression technique employed following the method of Pfaffl (2001)[27]. The primer sequence was shown in Table 1. PCR components include $1 \mu \mathrm{l}$ of forward primer, $1 \mu \mathrm{l}$ of revers primer, $5 \mu \mathrm{l}$ of cDNA template, $5.5 \mu \mathrm{l}$ RNAase free water and $12.5 \mu \mathrm{l}$ syber green mix. The total volume is $25 \mu \mathrm{l}$. Running $40 \mathrm{RT}-$ qPCR cycles according to the condition of kits then data were calculated using version 3.1 (StepOne ${ }^{\mathrm{TM}}$, USA).,software, The relative expression of P53 and androgen receptor was calculated using the comparative $\mathrm{Ct}$ method. The values were compared to beta actin genes and reported as fold change 
Table 1

Primers of targeted genes (P53 and AR ) with $\beta$ - actin

\begin{tabular}{|ll|}
\hline AR primer & $\begin{array}{l}\text { Forward primer, 5'-TGTGGTCAAGTGGGCCAAG-3'; } \\
\text { Reverse primer, 5'-TGCCATCTGGTCATCCACAT-3'. }\end{array}$ \\
\hline P53 primer & $\begin{array}{l}\text { Forward primer; 5'-CGCAAAAGAAGAAGCCACTA-3 } \\
\text { Reverse primer; 5'-TCCACTCTGGGCATCCTT-3 }\end{array}$ \\
\hline B- actin & $\begin{array}{l}\text { Forward primer; 5'-TGTTTGAGACCTTCAACACC-3' } \\
\text { Reverse primer; 5'-CGCTCATTGCCGATAGTGAT-3' }\end{array}$ \\
\hline
\end{tabular}

\subsection{Statistical analysis}

Results were analyzed using one-way analysis of variance (one way ANOVA) test and presented as mean \pm standard error (SEM), and followed by Tukey-Kramer methods for post hoc analysis. A value of $p<0.05$ was considered statistically significant.

\section{Results}

\subsection{Histopathological examination results:}

The histopathological examination of rat testis from the control group (Fig. 2a) indicated that the complete spermatogenesis; seminiferous tubules (ST) were lined by seprmatogonal cells, Sertoli cells with the presence of Leydig cells $(\mathrm{L})$ in the interstitial tissue. Testicular tissue of RJ group showed more or less normal seminiferous tubules having complete spermatogenesis (Fig. 2b). Examination of 6MP group revealed presence of severe testicular degeneration (vacuolation) $(V)$ in the tubular cells and necrosis (pyknosis) (Blue arrow) of seminiferous tubules (Fig. 2c) manifested by loss of tissue architecture and accompanied with accumulation of the degenerated cells within the lumen. Interstitial areas were widened with Leydig cells degeneration and presence of severe congestion (Black arrow). In $\mathrm{RJ}+6 \mathrm{MP}$ group, histologically, the testicular tissue showed variable degrees of improvement to the degree to be similar to that of control group (Fig. 2d)

\subsection{Effect of 6MP and RJ on testicular weights and epididymis length}

RJ alone gives the same results of safety such as control group without any significant differences, so they referred as control group.

Firstly we investigate the effects of $6 \mathrm{MP}$ alone and with RJ pre-treatment on testis weight, volume and tail of Epididymis of rats. Data in table (2) showed the significant decrease in testis weights, volume and in the length of epididymis in 6MP treated group at $\mathrm{P}<0.05$ compared to the control group. Prior 
administration of RJ in RJ + 6MP group evanished this decrement in compare to 6MP group by a significant degree at $\mathrm{P}<0.05$.

Table 2

The weights and volumes of testes and epididymis of different treated groups.

\begin{tabular}{|lllll|}
\hline \multicolumn{2}{|c}{ Testes } & \multicolumn{2}{c|}{ Tail of Epididymis } \\
\hline Groups & Weight $(\mathrm{g})$ & Volume $(\mathrm{cm} 3)$ & Weight $(\mathrm{g})$ & Volume $(\mathrm{cm} 3)$ \\
\hline Control group & $5.3 \pm 0.3$ & $4.76 \pm 0.01$ & $0.9 \pm 0.04$ & $2.8 \pm 0.21$ \\
\hline RJ group & $4.9 \pm 0.01$ & $4.5 \pm 0.06$ & $0.8 \pm 0.01$ & $2.6 \pm 0.01$ \\
\hline 6MP group & $3.01 \pm 0.02^{\mathrm{a}}$ & $2.12 \pm 0.02^{\mathrm{a}}$ & $0.3 \pm 0.01^{\mathrm{a}}$ & $0.91 \pm 0.03^{\mathrm{a}}$ \\
\hline RJ + 6MP group & $4.1 \pm 0.01^{\mathrm{b}}$ & $4.3 \pm 0.02^{\mathrm{b}}$ & $0.6 \pm 0.01^{\mathrm{b}}$ & $2.7 \pm 0.01^{\mathrm{b}}$ \\
\hline
\end{tabular}

The results were expressed as means $\pm S E n=10$. The data were analyzed by using one way ANOVA test. a: indicates a significant difference compared to control at level $p<0.05$. b: indicates a significant difference compared to $6 \mathrm{MP}$ at $\mathrm{P}<0.05$.

\subsection{Effect of 6MP and RJ on various sperm parameters in treated rats}

Administration of 6MP significantly decreased sperm motility \%, sperm count and sperm viability $\%$ at $\mathrm{P}<$ 0.05 when compared with control group. Also 6MP caused a significant increase of sperm abnormalities $\%$ when compared to control group at $\mathrm{P}<0.05$. In RJ + 6MP group; RJ caused a significant increase of sperm motility \%, sperm count and sperm viability \% with a concurrent reduction of sperm abnormalities $\%$ compared to $6 \mathrm{MP}$ group at $\mathrm{P}<0.05$. Semen analysis of RJ group showed no significant difference compared to control at $\mathrm{P}<0.05$.

Table 3

The changes in sperm motility, viability, abnormality percentage and sperm count in all examined rats

\begin{tabular}{|lllll|}
\hline Groups & Motility \% & Sperm cell count. $\left(\mathbf{X} \mathbf{1 0}^{6}\right)$ & Viability (\%) & Abnormalities (\%) \\
\hline Control group & $81.6 \pm 1$ & $30 \pm 2$ & $90.2 \pm 1.09$ & $15.9 \pm 0.9$ \\
\hline RJ group & $77.8 \pm 1.01$ & $31 \pm 0.1$ & $88.2 \pm 0.1$ & $16.3 \pm 0.3$ \\
\hline 6MP group & $40.2 \pm 0.5 \mathrm{a}$ & $15.9 \pm 0.4 \mathrm{a}$ & $50.5 \pm 1 \mathrm{a}$ & $42.1 \pm 0.5 \mathrm{a}$ \\
\hline RJ + 6MP group & $79.9 \pm 1 \mathrm{~b}$ & $29.32 \pm 1 \mathrm{~b}$ & $88.12 \pm 1.2 \mathrm{~b}$ & $16.5 \pm 1 \mathrm{~b}$ \\
\hline
\end{tabular}

The results were were analyzed by using one way ANOVA test and expressed as means $\pm S E, n=10$. The data. a: indicates a significant difference compared to control at level $p<0.05$. b: indicates a significant 


\subsection{Effect of 6MP and RJ on testicular AR mRNA expression and serum testosterone, FSH and LH concentrations}

As shown in table (3); AR mRNA significantly decreased in 6MP group at $\mathrm{P}<0.05$ compared to control group, while pre-administration of RJ prevent the negative effect of 6MP on AR mRNA that revealed in significant increase of AR mRNA in RJ + 6MP group at $P<0.05$ compared to 6MP group.

$6 \mathrm{MP}$ treated rats showed a significant increase of FSH, $\mathrm{LH}$ and a significant decrease of testosterone level at $\mathrm{P}<0.05$, compared to control group. The levels of FSH and LH of RJ $+6 \mathrm{MP}$ group were significantly decreased and serum testosterone level was significantly increased at $P<0.05$ when compared to 6MP group. The levels of AR mRNA expression and hormonal levels of RJ group and control are not significant at $P<0.05$.

Table 3. The effect of 6MP on AR mRNA expression (fold change in relation to $\beta$-actin), testosterone, FSH and $\mathrm{LH}$ concentrations in different treated groups.

The results were expressed as means \pm SE $n=10$. The data were analyzed by using one way ANOVA test. a: indicates a significant difference compared to control at level $p<0.05$. b: indicates a significant difference at $\mathrm{P}<0.05$.

\subsection{Effects of 6MP and RJ on testicular antioxidant/ oxidative redox.}

The results in Fig. 3 depicted that administration of 6MP disturbed the testicular antioxidant/oxidative redox and caused a significant testicular oxidative stress. This effect was indicated by the ability of $6 \mathrm{MP}$ to cause a significant depletion of testicular GSH concentration, GR, Catalase and SOD activities with a concurrent increase of MDA level in 6MP group as compared with control group at $P<0.05$. In $R J+6 M P$ group; pretreatment of RJ restored the testicular antioxidant/ oxidative redox. This protective effect was indicated by the significant increase of GSH concentration, GR, Catalase, SOD activities and the significant reduction of MDA level in RJ +6MP group compared to 6MP group at $\mathrm{P}<0.05$. In addition, testicular antioxidant/oxidative redox of RJ group showed no significant difference compared to control group at $\mathrm{P}<0.05$.

\subsection{The effect of 6MP and RJ on testicular PI3K, AKT, Nrf2 and $\mathrm{HO1}$ of different treated groups}

Western blotting results (Fig. 3) showed that administration of 6MP significantly decreased the expression of testicular PI3K, AKT, Nrf2 and HO1 protein levels in 6MP group compared to control group 
at $\mathrm{P}<0.05$. RJ significantly increased $\mathrm{PI} 3 \mathrm{~K}, \mathrm{AKT}$, Nrf2 and $\mathrm{HO} 1$ protein levels in RJ +6MP group compared to $6 \mathrm{MP}$ at $\mathrm{P}<0.05$. PI3K and AKT protein levels in RJ +6MP group were significantly lower than control group at $\mathrm{P}<0.05$ while Nrf2 and $\mathrm{HO} 1$ protein levels of RJ +6MP group showed no significant difference as compared to control group at $\mathrm{P}<0.05$.

\subsection{The effect of 6MP and RJ on TNF-a, DNA fragmentation\%, P 53 mRNA expression and caspase-3 of different treated groups.}

Results in Fig. 4 showed that 6MP significantly increased TNF-a, DNA fragmentation \%, P53 mRNA expression and caspase-3 in 6MP group compared to control group at $\mathrm{P}<0.05$. RJ administration reduced testicular inflammatory marker, DNA damage and apoptosis. This effect was indicated by the significant reduction of TNF-a, DNA fragmentation \%, P53 and caspase-3 respectively in RJ + 6MP group compared to 6MP group at $\mathrm{P}<0.05$.TNF-a and DNA fragmentation \% of RJ + 6MP group were higher than those in control group at $P<0.05$. There was no significant difference between P53 and caspase-3 of RJ $+6 \mathrm{MP}$ and control group at $\mathrm{P}<0.05$.

\section{Discussion}

$6 \mathrm{MP}$ is an active metabolite of azathioprine, clinically used in chemotherapy and immunosuppression. Despite, the efficacy of $6 \mathrm{MP}$ in treatment, it has cytotoxic effect on normal body tissues $[28,29]$. Our study insight into 6MP gonadotoxicity and infertility problems and the effect of RJ as a prophylactic agent through studying changes in sperm parameters and seminiferous tubules.

It is reported that the active metabolites of 6MP damages rapidly dividing cells, such as those in the bone marrow, intestinal epithelium, and reproductive organs of adults [30]. The reduction of the testicular and epididymal weight in 6MP treated rats indicated its obvious gonadotoxic effect. This effect was confirmed by the histological study of testicular tissues which showed a severe atrophy of many seminiferous tubules with wide interstitial spaces, few numbers of spermatozoa and spermatogenic cells, also morphology distortion and atrophy of Leydig cells. In agreement with our results, Karawya et.al; reported that azathioprine induce testicular atrophy, histological distortion and impair spermatogenesis in male albino rats [31].

Statistical analysis of semen parameters in 6MP treated rats revealed a severe reduction of sperm count, motility and viability, as well as an increase in abnormal forms of spermatozoa. This effect of 6MP could be explained by the ability of $6 \mathrm{MP}$ to interfere with nucleic acid synthesis of rapidly dividing somatic and germ cells resulting in germ cell apoptosis and germ cell mutations [32].

Our data clarify that 6MP treatment significantly decreased gene expression of ARs in 6MP treated rats. ARs are nuclear receptors highly expressed in testis and play a crucial role in spermatogenesis 
maintenance. The reduction of ARs and their knockout alters the reproductive development and spermatogenesis [33].

Spermatogenesis is highly susceptible to reactive oxygen species (ROS) levels, which increases spermatogenic cells apoptosis and induces seminiferous epithelium damage [34], testicular dysfunction and sperm abnormalities [35]. In the metabolic process of 6MP, xanthine oxidase converts 6MP to thiouric acid, a reaction capable of producing ROS, almost $\mathrm{H}_{2} \mathrm{O}_{2}$ [36]. We demonstrated that $6 \mathrm{MP}$ alters testicular antioxidant/ oxidative redox by decreasing testicular antioxidants; GSH concentration, GR, catalase and SOD activities with a concurrent increase of testicular lipid peroxidation by increasing MDA level. Protein concentration of Nrf2 and H01 were significantly decreased in 6MP treated rats. Türk et al., (2010) proved the correlation between oxidative stress and sperm abnormalities [37]. In consistent with these reports our results showed that reduction of antioxidants was associated with DNA fragmentation and was accompanied by significant increased percentages of abnormal, dead and immotile sperms in 6MP treated rats

Elevation of reactive oxygen species (ROS) is associated with Testicular inflammation, 6MP induced inflammation was evidenced through histomorphological changes of testicular tissues and elevated levels of TNF-a in testicular homogenate. TNF-a, produced by testis germ cells and Sertoli cells, is an essential cytokine that regulates multiple cellular processes in testis [38]. The susceptibility of spermatogenesis to TNF-a has been reported [39], our data clarify that significant increase in TNF-a in $6 \mathrm{MP}$ treated rats has associated with increasing abnormal forms of sperms and reduction in sperm viability. TNF- $a$ in addition of being mediator of inflammation it also induces apoptosis [40-42].

To insight into 6MP-induced testicular apoptosis, we evaluated PI3K and AKT protein expression, proapoptotic caspase-3 activity and mRNA expression of P53. PI3K/AKT signaling pathway plays a pivotal role in cell apoptosis, proliferation and differentiation [43]. In addition, PI3K/AKT is related to the growth and development of testicular tissue and is involved in the interaction between FSH and Sertoli cells [44]. PI3Ks are a family of important lipid kinases. PI3K can be activated by cell surface receptor [45] and it can act as a messenger molecule involved in signal transduction which is the first regulator of AKT activation. The activated PI3K1 can make AKT transpose to the cell membrane and activate it. AKT, a serine-threonine kinase, is an important downstream protein of PI3K. AKT mainly exists in three forms: AKT1, AKT2 and AKT3. AKT1 is the main form in testis which located in spermatogenic cells and Sertoli cells. In accordance with our findings, Reggio et al., (2017), report that 6MP down regulated PI3K/AKT pathway [46].

Down-regulation of PI3K/AKT pathway can results in activation of caspase-3 and trigger apoptosis[47]. In support to the previous report this study illustrated that administration of 6MP for 20 days induced downregulation of PI3K and AKT which was associated with high activity of caspase- 3 and over expression of P53 in 6MP treated rats. In confirm to our result Kanemitsu, et al.,(2009) reported that 6MP induced apoptosis through P53, caspase-3 pathway [48]. 
It is reported that the overexpression of caspase-3 is associated with Leydig cell atrophy in 6MP treated rats [6]. Leydig cells are decisive to production of testosterone, which can effect on function of Sertoli cells. Atrophy of Leydig cells results in reduction of testosterone levels that also associated with elevated values of FSH and LH. Therefore premature detachment of spermatids may occur due to decrement in testosterone levels. Due to the essential role of testosterone in spermatogenesis, reduction of its level was associated with sperm abnormalities and reduced sperm count that was associated with Sertoli cell dysfunction, and impairment of spermatogenesis[31, 49]. Leydig cells atrophy in 6MP treated rats was accompanied by significant decrease in serum testosterone levels in compare to other groups

Herein, we evidenced the ameliorative effects of RJ in 6MP-induced testicular dysfunction and the ability of RJ to improve spermatogenesis process in RJ pretreated rats. The data of RJ + 6MP clearly revealed a significant increase in testicular weight, sperm count, motility and viability as well as reduction in abnormal forms of sperms which reversed the impairment occurred in 6MP treated rats. The improvement of histomorphological data of RJ pretreated rats evidenced the ability of RJ for tissue repair and protection against tissue damage, in accordance with our finding Temamoğulları, et.al, (2018) report the protective effect of RJ against mice testicular degeneration and spermiotoxicity induced by flunixin [11].

Pre-administration of RJ increase serum testosterone and reduced the concentration of FSH and $\mathrm{LH}$ in $\mathrm{RJ}+6 \mathrm{MP}$ group, RJ ability to increase serum testosterone levels might be due to Zn containing which has an essential role in spermatogenesis [50]

Antioxidant activity of RJ has been reported [51-53]. In agreement with previous reports we evidenced that RJ pretreatment restored levels of SOD, catalase, GR and GSH to approach normal control levels and scavenged ROS, therefore reduced lipid peroxidation (low MDA level). The ability of RJ to scavenge the ROS might be due to RJ up-regulation of Nrf2 expression which increased significantly in RJ pretreated rats with concurrent increase in $\mathrm{HO}-1$ protein levels in compare with 6MP treated rats. in consistence with our data Almeer, et al (2018) illustrate that RJ abrogated the testicular oxidative stress induced by cadmium through up-regulation of Nrf2 [54]. Nrf2 up regulation stimulates the expression of HO-1, upregulation of Nrf2/H01 pathway increases antioxidants activity and protects against tissue [55] damages, in agreement with this report our histopathological finding of RJ +6MP revealed markedly improvement in seminiferous tubules appearance as well as Leydig cell numbers in compare with 6MP group. In addition to antioxidant activity of RJ, it has anti-inflammatory effects, Kohno, et al reported the inhibitory effect of RJ on pro-inflammatory cytokines by activation of macrophages [56], also report of Aslan, Z, et al who illustrated that RJ abrogated renal inflammation induced by ethylene glycol [57]. In the present study we found that rats which pretreated with RJ revealed markedly decreased TNF-a activity when compared with 6MP treated rats, this result evidenced the inhibitory effect of RJ on TNF- a which was associated with spermatogenesis enhancement.

Anti-apoptotic effect of RJ is supported by report of Karadeniz, A., et al., (2011) who suggest that RJ acts as anti-apoptotic agent and antioxidant when used in co- administration with cisplatin in kidney and liver 
[58]. In addition Valiollahpoor Amiri, et al (2016) report that RJ inhibit apoptosis by increasing the expression of BCL2 [59]. In present study we insight into the mechanistic pathway of RJ anti-apoptotic effect through following PI3K/AKT pathway and pro-apoptotic caspase-3 with deepen in P53 mRNA gene expression. Pre-administration of RJ induced up-regulation of PI3K/AKT pathway and caspase-3 inhibition. Data of RJ pretreated rats revealed that high protein levels of PI3K and AKT was associated with decrement in caspase-3 activity and P53 mRNA expression. In addition, histopathological data of RJ $+6 \mathrm{MP}$ appeared to restore normal number of Leydig cells and seminiferous tubule appearance that approach control data. Normal size and number of Leydig cells was observed in RJ pretreatment also testosterone levels was restored to approach normal control levels. The positive impact of RJ on Leydig cells could be due to suppression of caspase-3 activity, [6] reported that caspase-3 activity induced Leydig cell death .The possible mechanism of RJ protection against 6MP-induced apoptosis is the upregulation of PI3K/AKT pathway and inhibition of caspase-3 activity. A recent study proved that $\mathrm{PI3K} / \mathrm{AKT}$ pathway correlates with Nrf2 expression in testicular tissue and regulates the level of Nrf2dependent inducible expression of HO-1 [60]. In support with the previous report, we reported that RJ upregulation of PI3K/AKT pathway was concurrent with increasing in Nrf2 protein levels in RJ pretreated rats.

Finally, the present study proves that Royal Jelly is a safe product for albino rats and can be used in combination with $6 \mathrm{MP}$ to reduce or mitigate $6 \mathrm{MP}$ toxicity on testicular tissues through up-regulation of PI3K/AKT pathway, Nrf2/HO-1 pathway and down regulation of caspase-3, P53 gene expression and TNF-a. In confirm to our results Ahmed, W.M., et al., report that RJ prevent hepatotoxicity induced by azathioprine [13]. RJ acts as scavenger to ROS therefore decrease DNA fragmentation percent, increase antioxidants activity and decrease peroxidation levels in testicular tissues. The efficacy of RJ as antiinflammatory, antioxidant and anti-apoptotic agent qualify it to attenuate chemotherapy induced toxicity and prevent testicular dysfunction caused by $6 \mathrm{MP}$

\section{Conclusion}

The present study demonstrates that RJ protects 6MP-induced testicular injury via regulating PI3K/AKT signaling pathway. Therefore, RJ might be a promising treatment for infertility diseases related to testicular injuries.

\section{Declarations}

\section{Acknowledgment}

The authors thank the Laboratory of biochemistry and chemistry of nutrition, the Department of Pathology of faculty of veterinary medicine, Beni-Suef University for their assistance.

Authors' contributions: KS and AZ designed the study and prepared the manuscript. KS and NW performed the experiments. KS, AZ and NW assisted in the data analysis and helped prepare the 
manuscript. All authors read and approved the final manuscript.

Funding: authors declare that they haven't received any kind of financial support.

\section{Availability of data and materials:}

The datasets used and/or analyzed during the current study available from the corresponding author on reasonable request.

\section{Ethics approval and consent to participate:}

All procedures were approved by the Committee for the Ethics on Animal Care and Experiments of BeniSuef University.

Consent for publication: All authors agree to the submission of this article for publication.

Competing interests: The authors declare that they have no competing interests.

\section{Author details:}

1: Biochemistry Department, Faculty of Veterinary Medicine, Beni-Suef University, Egypt. 2: Biotechnology and Life Sciences Department, Faculty of Postgraduate Studies for Advanced Sciences, Beni-Suef University, Egypt. Corresponding author: Khalid Shaaban Hashem (K. S. Hashem)

khalidshaabanhashem@yahoo.com Contact/ Address: Biochemistry department, Faculty of veterinary medicine, Beni-Suef University, Beni-Suef 62511, Egypt.

\section{References}

1. Whalen, K., Lippincott illustrated reviews: pharmacology. 2018: Lippincott Williams \& Wilkins.

2. Stuckert, A.J., et al., Use of allopurinol to reduce hepatotoxicity from 6-mercaptopurine (6-MP) in patients with acute lymphoblastic leukemia (ALL). Leukemia \& Lymphoma., 2019: p. 1-4.

3. Mohammadi, O. and T.A. Kassim, Azathioprine, in StatPearls [Internet]. 2019, StatPearls Publishing.

4. Wu, Y.T., et al., Azathioprine hepatotoxicity and the protective effect of liquorice and glycyrrhizic acid. An International Journal Devoted to Pharmacological and Toxicological Evaluation of Natural Product Derivatives, 2006. 20(8): p. 640-645.

5. Bendre, S.V., et al., Lymphocyte Hprt mutant frequency and sperm toxicity in C57BL/6 mice treated chronically with Azathioprine. Mutation Research/Fundamental and Molecular Mechanisms of Mutagenesis, 2005. 578(1-2): p. 1-14.

6. Morgan, J.A., et al., Apoptosome activation, an important molecular instigator in 6-mercaptopurine induced Leydig cell death. Scientific reports, 2015. 5: p. 16488. 
7. Golmohammadi, M.G., et al., Protective Effect of Gallic Acid on Testicular Tissue, Sperm Parameters, and DNA Fragmentation against Toxicity Induced by Cyclophosphamide in Adult NMRI Mice. Urology journal, 2019.

8. Nagai, T. and R.J.F.c. Inoue, Preparation and the functional properties of water extract and alkaline extract of royal jelly. Food chemistry, 2004. 84(2): p. 181-186.

9. Silici, S., et al., Antioxidative effect of royal jelly in cisplatin-induced testes damage. Urology, 2009. 74(3): p. 545-551.

10. Ghanbari, E., V. Nejati, and M.J.I.J.o.R.B. Khazaei, Antioxidant and protective effects of Royal jelly on histopathological changes in testis of diabetic rats. International Journal of Reproductive BioMedicine, 2016. 14(8): p. 519.

11. Temamoğulları, F., F. Aral, and R.J.P.j.o.v.s. Yılmaz, Royal jelly protection on flunixin meglumineinduced spermiotoxicity and testicular degeneration in mice. Polish journal of veterinary sciences, 2018.

12. Jalali, A.S., et al., Royal jelly alleviates sperm toxicity and improves in vitro fertilization outcome in stanozolol-treated mice. Iranian journal of reproductive medicine, 2015. 13(1): p. 15.

13. Ahmed, W.M., et al., Royal jelly attenuates azathioprine induced toxicity in rats. Environmental toxicology and pharmacology, 2014. 37(1): p. 431-437.

14. Parasuraman, S., R. Raveendran, and R. Kesavan, Blood sample collection in small laboratory animals. Journal of pharmacology \& pharmacotherapeutics, 2010. 1(2): p. 87.

15. Ahmed, M.A.J.T. and a. pharmacology, Amelioration of nandrolone decanoate-induced testicular and sperm toxicity in rats by taurine: effects on steroidogenesis, redox and inflammatory cascades, and intrinsic apoptotic pathway. 2015. 282(3): p. 285-296.

16. Wyrobek, A.J., et al., An evaluation of the mouse sperm morphology test and other sperm tests in nonhuman mammals: A report of the US Environmental Protection Agency Gene-Tox Program. Mutation Research/Reviews in Genetic Toxicology, 1983. 115(1): p. 1-72.

17. Sönmez, M., G. Türk, and A.J.T. Yüce, The effect of ascorbic acid supplementation on sperm quality, lipid peroxidation and testosterone levels of male Wistar rats. Theriogenology, 2005. 63(7): p. 20632072.

18. Suvarna, K.S., C. Layton, and J.D. Bancroft, Bancroft's Theory and Practice of Histological Techniques E-Book. 2018: Elsevier Health Sciences.

19. Ohkawa, H., N. Ohishi, and K.J.A.b. Yagi, Assay for lipid peroxides in animal tissues by thiobarbituric acid reaction. Analytical biochemistry, 1979. 95(2): p. 351-358.

20. Aebi, H., [13] Catalase in vitro, in Methods in enzymology. 1984, Elsevier: Academic Press. p. 121-126.

21. Koracevic, D., et al., Method for the measurement of antioxidant activity in human fluids. J Clin Pathol, 2001. 54(5): p. 356-61.

22. Beutler, E., et al., Colorimetric method for determination of glutathione reductase concentration. Journal of Laboratory and Clinical Medicine, 1963. 61: p. 882. 
23. Brigelius, R., et al., Identification and quantitation of glutathione in hepatic protein mixed disulfides and its relationship to glutathione disulfide. Biochemical pharmacology., 1983. 32(17): p. 2529-2534.

24. Berry, M., et al., TNF-a in asthma. Current opinion in pharmacology, 2007. 7(3): p. 279-282.

25. Collins, J.A., et al., Major DNA fragmentation is a late event in apoptosis. Journal of Histochemistry \& Cytochemistry, 1997. 45(7): p. 923-934.

26. Weber, K. and M.J.J.o.B.C. Osborn, The reliability of molecular weight determinations by dodecyl sulfate-polyacrylamide gel electrophoresis. Journal of Biological Chemistry, 1969. 244(16): p. 44064412.

27. Pfaffl, M.W.J.N.a.r., A new mathematical model for relative quantification in real-time RT-PCR. Nucleic acids research, 2001. 29(9): p. e45-e45.

28. Clark, P., Y. Hsia, and R.J.B.m.j. Huntsman, Toxic complications of treatment with 6-mercaptopurine. British medical journal, 1960. 1(5170): p. 393.

29. Tapner, M.J., et al., Toxicity of low dose azathioprine and 6-mercaptopurine in rat hepatocytes. Roles of xanthine oxidase and mitochondrial injury. Journal of hepatology., 2004. 40(3): p. 454-463.

30. Bendre, S.V., et al., Effect of chronic azathioprine treatment on germ-line transmission of Hprt mutation in mice. Environmental and Molecular mutagenesis, 2007. 48(9): p. 744-753.

31. Karawya, F.S. and A.F.J.C.T. El-Nahas, The protective effect of vitamin C on Azathioprine induced seminiferous tubular structural changes and cytogenetic toxicity in albino rats. Cancer Ther, 2006. 4: p. 125-34.

32. Brown, S.M., et al., Systemic medications used in treatment of common dermatological conditions: safety profile with respect to pregnancy, breast feeding and content in seminal fluid. Journal of Dermatological Treatment, 2019. 30(1): p. 2-18.

33. Wang, R.-S., et al., Androgen receptor roles in spermatogenesis and fertility: lessons from testicular cell-specific androgen receptor knockout mice. Endocrine reviews, 2009. 30(2): p. 119-132.

34. Moazamian, R., et al., Oxidative stress and human spermatozoa: diagnostic and functional significance of aldehydes generated as a result of lipid peroxidation. MHR: Basic science of reproductive medicine, 2015. 21(6): p. 502-515.

35. Pahune, P.P., et al., The total antioxidant power of semen and its correlation with the fertility potential of human male subjects. Journal of clinical and diagnostic research: JCDR. , 2013. 7(6): p. 991.

36. Kelley, E.E., et al., Hydrogen peroxide is the major oxidant product of xanthine oxidase. Free Radical Biology and Medicine, 2010. 48(4): p. 493-498.

37. Türk, G., et al., Attenuation of cyclosporine A-induced testicular and spermatozoal damages associated with oxidative stress by ellagic acid. International Immunopharmacology., 2010. 10(2): p. 177-182.

38. Mruk, D.D. and C.Y.J.E.r. Cheng, Sertoli-Sertoli and Sertoli-germ cell interactions and their significance in germ cell movement in the seminiferous epithelium during spermatogenesis. Endocrine reviews, 2004. 25(5): p. 747-806. 
39. Ramonda, R., et al., Influence of tumor necrosis factor a inhibitors on testicular function and semen in spondyloarthritis patients. Fertility and sterility, 2014. 101(2): p. 359-365.

40. Chen, G. and D.V.J.S. Goeddel, TNF-R1 signaling: a beautiful pathway. Science, 2002. 296(5573): p. 1634-1635.

41. Rath, P.C. and B.B.J.J.o.c.i. Aggarwal, TNF-induced signaling in apoptosis. Journal of clinical immunology, 1999. 19(6): p. 350-364.

42. Ségui, B., et al., Involvement of FAN in TNF-induced apoptosis. The Journal of clinical investigation, 2001. 108(1): p. 143-151.

43. Franke, T.J.O., PI3K/Akt: getting it right matters. Oncogene, 2008. 27(50): p. 6473.

44. Walker, W.H. and J.J.R. Cheng, FSH and testosterone signaling in Sertoli cells. Reproduction, 2005. 130(1): p. 15-28.

45. Lee, S., et al., Signaling pathways of bisphenol A-induced apoptosis in hippocampal neuronal cells: Role of calcium-induced reactive oxygen species, mitogen-activated protein kinases, and nuclear factor-KB. Journal of neuroscience research, 2008. 86(13): p. 2932-2942.

46. Reggio, A., et al., The immunosuppressant drug azathioprine restrains adipogenesis of muscle Fibro/Adipogenic Progenitors from dystrophic mice by affecting AKT signaling. Scientific reports, 2019. 9(1): p. 4360.

47. Wang, H., et al., Role of PISK/AKT/mTOR signaling pathway in DBP-induced apoptosis of testicular sertoli cells in vitro. Environmental toxicology and pharmacology, 2017. 53: p. 145-150.

48. Kanemitsu, $\mathrm{H}$., et al., 6-mercaptopurine (6-MP) induces p53-mediated apoptosis of neural progenitor cells in the developing fetal rodent brain. Neurotoxicology and teratology, 2009. 31(4): p. 198-202.

49. Kumar, S.G., et al., Dacarbazine induces genotoxic and cytotoxic germ cell damage with concomitant decrease in testosterone and increase in lactate dehydrogenase concentration in the testis. Mutation Research/Genetic Toxicology, 2006. 607(2): p. 240-252.

50. Fallah, A., et al., Zinc is an essential element for male fertility: A review of zn roles in men's health, germination, sperm quality, and fertilization. Journal of reproduction \& infertility, 2018. 19(2): p. 69.

51. Delkhoshe-Kasmaie, F., et al., Royal jelly protects from taxol-induced testicular damages via improvement of antioxidant status and up-regulation of E2f1. Systems biology in reproductive medicine., 2014. 60(2): p. 80-88.

52. Ghanbari, E., et al., Study on the effect of royal jelly on reproductive parameters in streptozotocininduced diabetic rats. International journal of fertility \& sterility., 2015. 9(1): p. 113.

53. Najafi, G., et al., Protective role of royal jelly in oxymetholone-induced oxidative injury in mouse testis. Iranian Journal of Toxicology, 2014. 8(25).

54. Almeer, R., et al., Royal Jelly Abrogates Cadmium-Induced Oxidative Challenge in Mouse Testes: Involvement of the Nrf2 Pathway. International journal of molecular sciences, 2018. 19(12): p. 3979.

55. Bae, W.J., et al., Protective effect of decursin extracted from Angelica gigas in male infertility via Nrf2/HO-1 signaling pathway. Oxidative Medicine and Cellular Longevity, 2016. 2016. 
56. Kohno, K., et al., Royal jelly inhibits the production of proinflammatory cytokines by activated macrophages. Bioscience, biotechnology,and biochemistry, 2004. 68(1): p. 138-145.

57. Aslan, Z. and L.J.I.b.j.u. Aksoy, Anti-inflammatory effects of royal jelly on ethylene glycol induced renal inflammation in rats. International braz j urol, 2015. 41(5): p. 1008-1013.

58. Karadeniz, A., et al., Royal jelly modulates oxidative stress and apoptosis in liver and kidneys of rats treated with cisplatin. Oxidative medicine and cellular longevity, 2011. 2011.

59. Valiollahpoor Amiri, M., H. Deldar, and Z.J.S.b.i.r.m. Ansari Pirsaraei, Impact of supplementary royal jelly on in vitro maturation of sheep oocytes: genes involved in apoptosis and embryonic development. Systems biology in reproductive medicine, 2016. 62(1): p. 31-38.

60. Bashir, N., et al., The molecular and biochemical insight view of grape seed proanthocyanidins in ameliorating cadmium-induced testes-toxicity in rat model: implication of PI3K/Akt/Nrf-2 signaling. Bioscience reports, 2019. 39(1): p. BSR20180515.

\section{Figures}

\section{Control group $\mid \_\quad$ rats received $0.9 \%$ saline 0 day \\ 30th day}

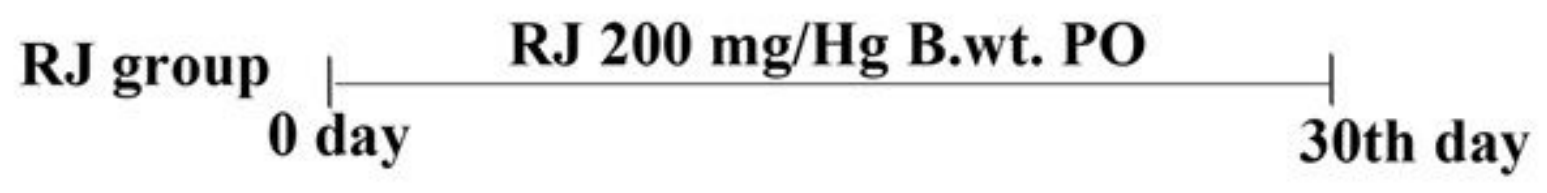

6-MP group $\mid$ Saline PO $\mid$ 6-MP $5 \mathrm{mg} / \mathrm{kg}$ PO daily 0 day 10th day 30th day

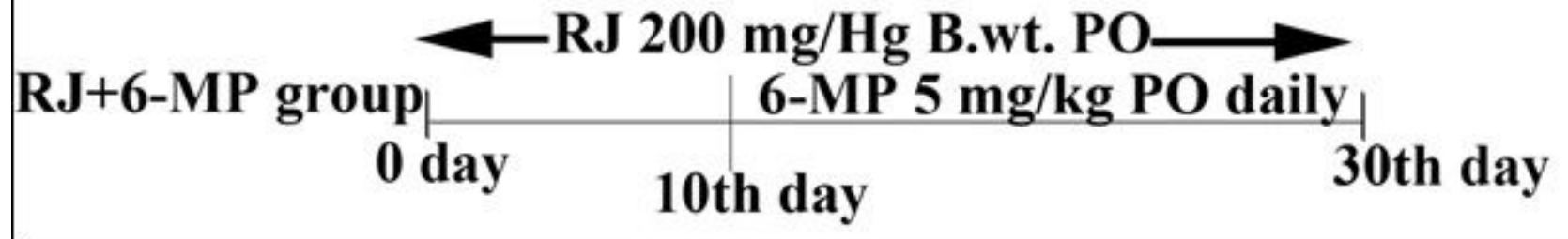

Figure 1

Summary of the experimental design shows the different treated groups, specific dose and duration of each treatment. 

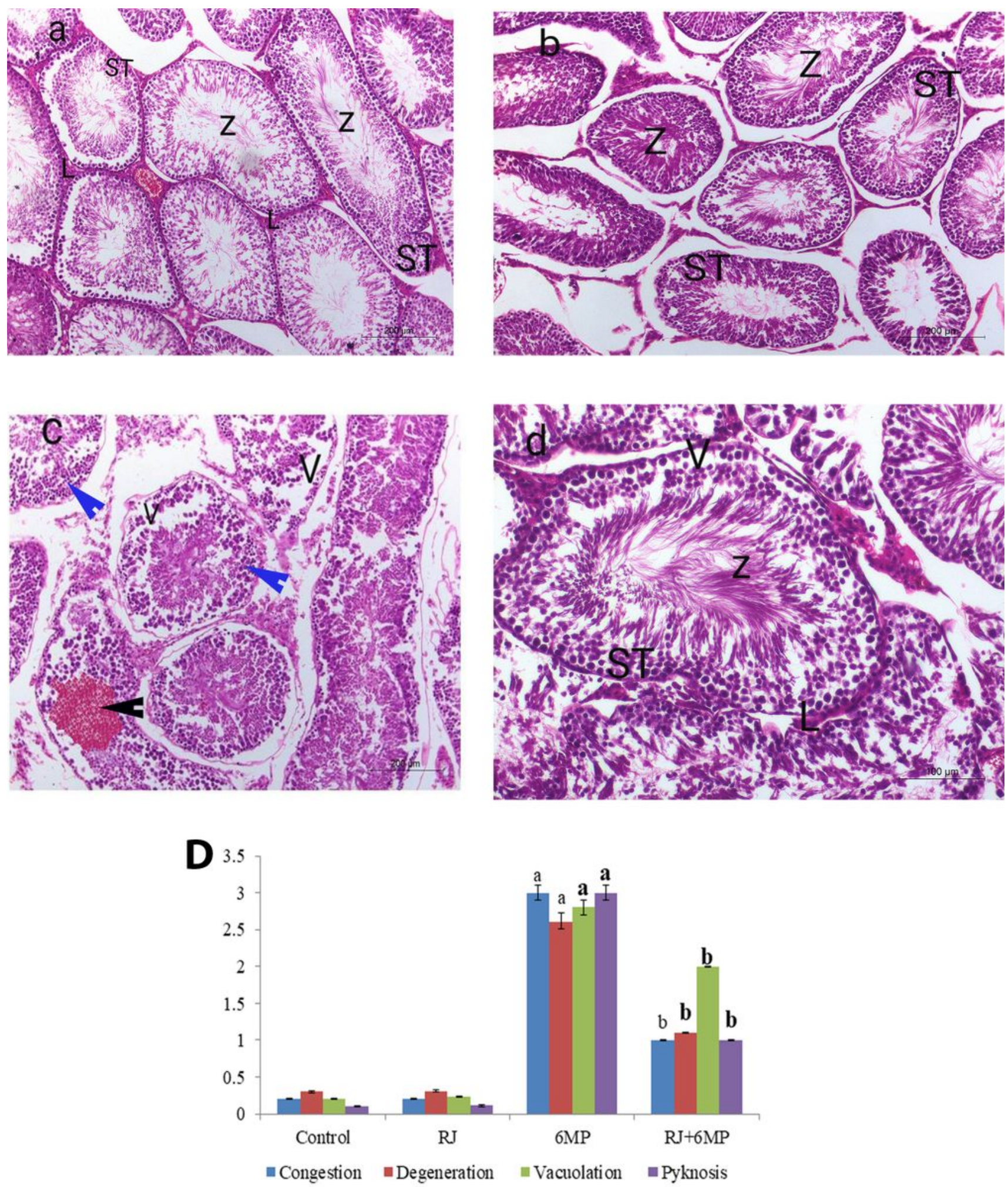

Figure 2

Representative photomicrographs of H\&E-stained sections of rat testes in each experimental group: a) Light microscopy of the testicular tissue of control group showed a complete spermatogenesis; seminiferous tubules (ST) were lined by seprmatogonal cells, Sertoli cells with the presence of Leydig cells $(L)$ in the interstitial tissue, $(Z)$ spermatozoa. b) The testicular tissue of RJ group showed more or less normal seminiferous tubules having complete spermatogenesis. c) The testicular tissue of 6MP 
group revealed the presence of severe testicular degeneration; vacuolation $(V)$ in the tubular cells and pyknosis (Blue arrow) of seminiferous tubules, loss of tissue architecture and accompanied with accumulation of the degenerated cells within the lumen. Interstitial areas were widened with degenerated Leydig cells and presence of severe congestion (Black arrow). d) teaticular tissue of RJ+6MP group showed variable degrees of improvement to the degree to be similar to that of control group The histopathological examination of rat testis from the control group (fig.2a) indicated that (fig.2b). (fig. 2d)
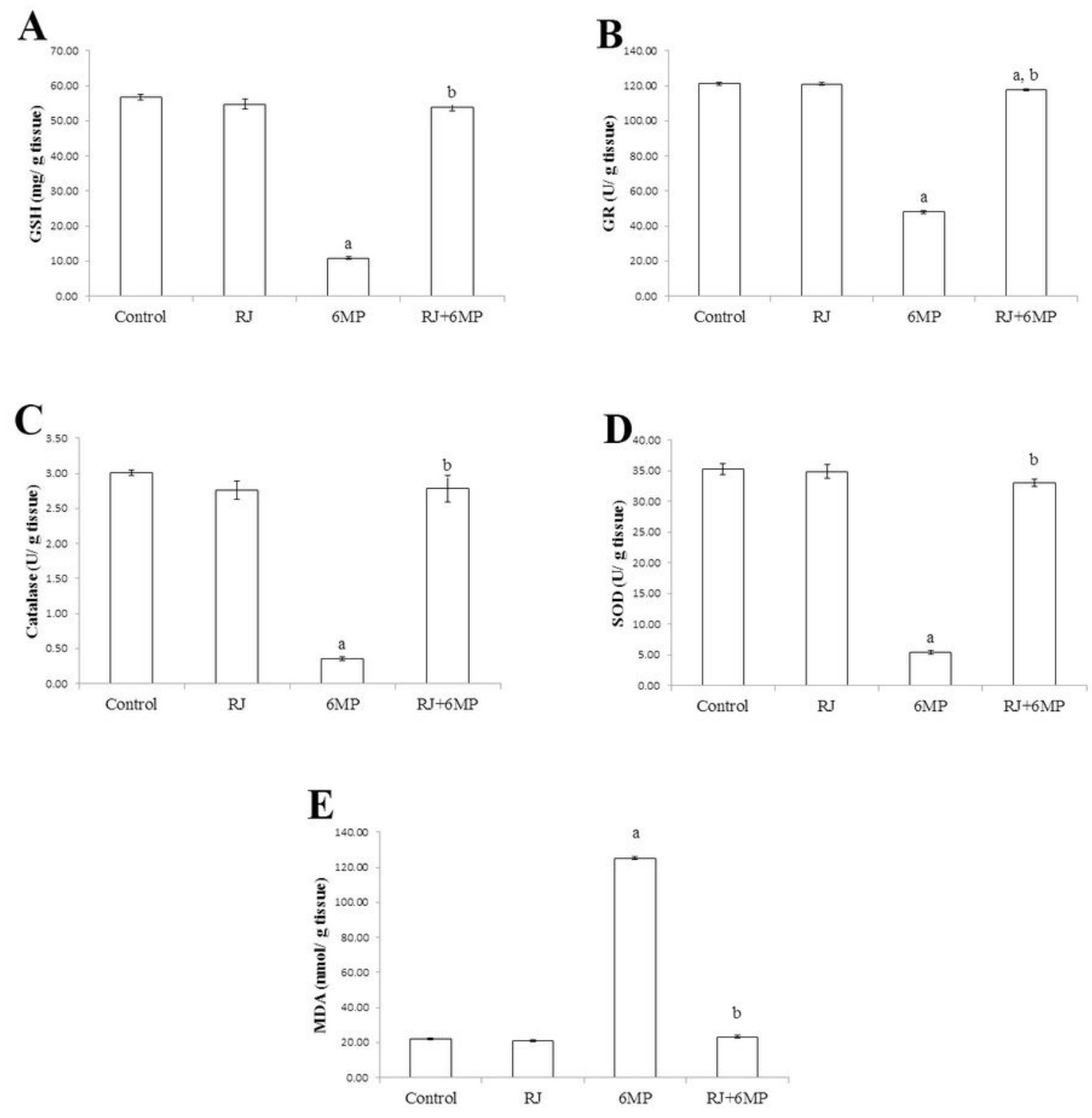

Figure 3 
Testicular antioxidant/oxidative redox parameters. A) GSH; reduced glutathione (mg/ g tissue). B) GR; glutathione reductase (U/g tissue), C) Catalase (U/g tissue), D) SOD; super oxide dismutase (U/ g tissue) and $\mathrm{E}) \mathrm{MDA}$; malonaldhyde ( $\mathrm{nmol} / \mathrm{g}$ tissue). Values are means $\pm \mathrm{SE}, \mathrm{n}=10$. a indicates a significant difference compared to control group at $\mathrm{P}<0.05$. $\mathrm{b}$ indicates a significant difference compared to $6 \mathrm{MP}$ at $\mathrm{P}<0.05$.

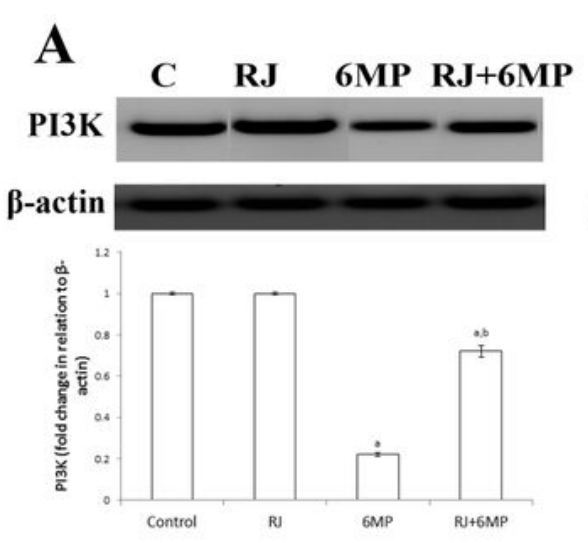

B

\section{AKT}

C RJ 6MP RJ+6MP

$\beta$-actin
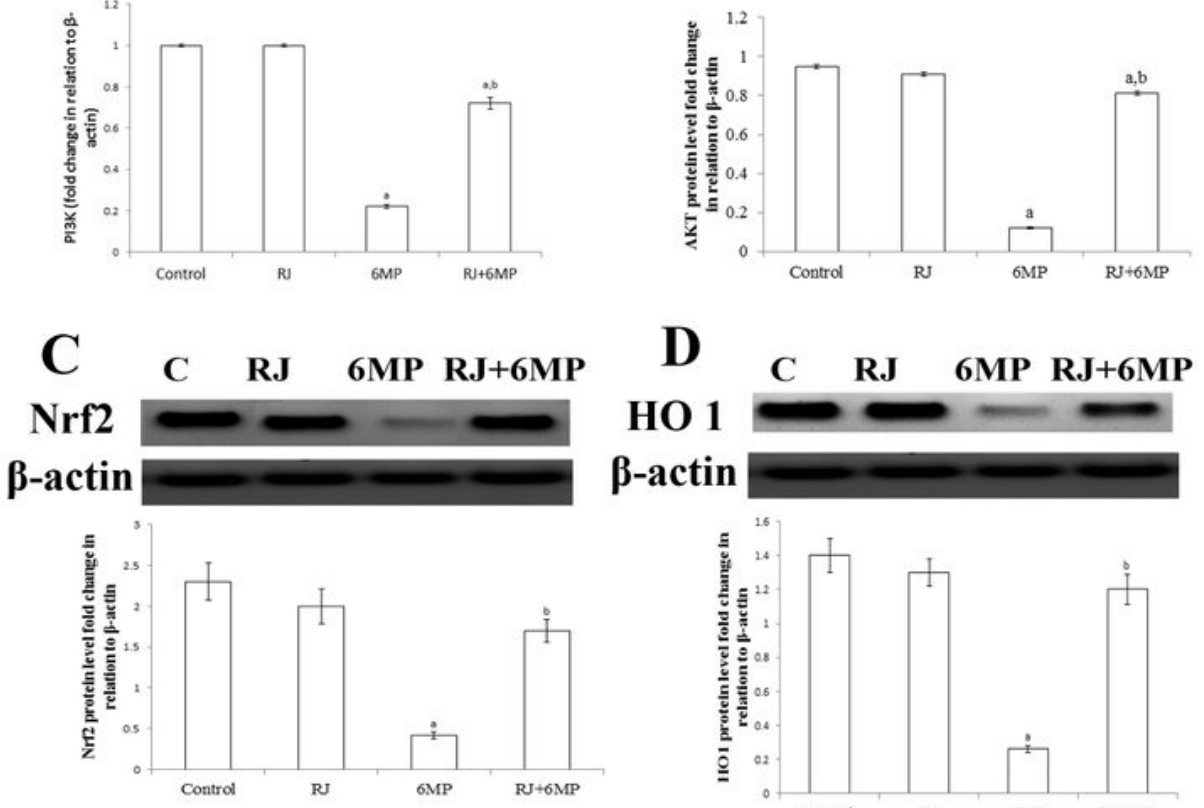

$\beta$-actin
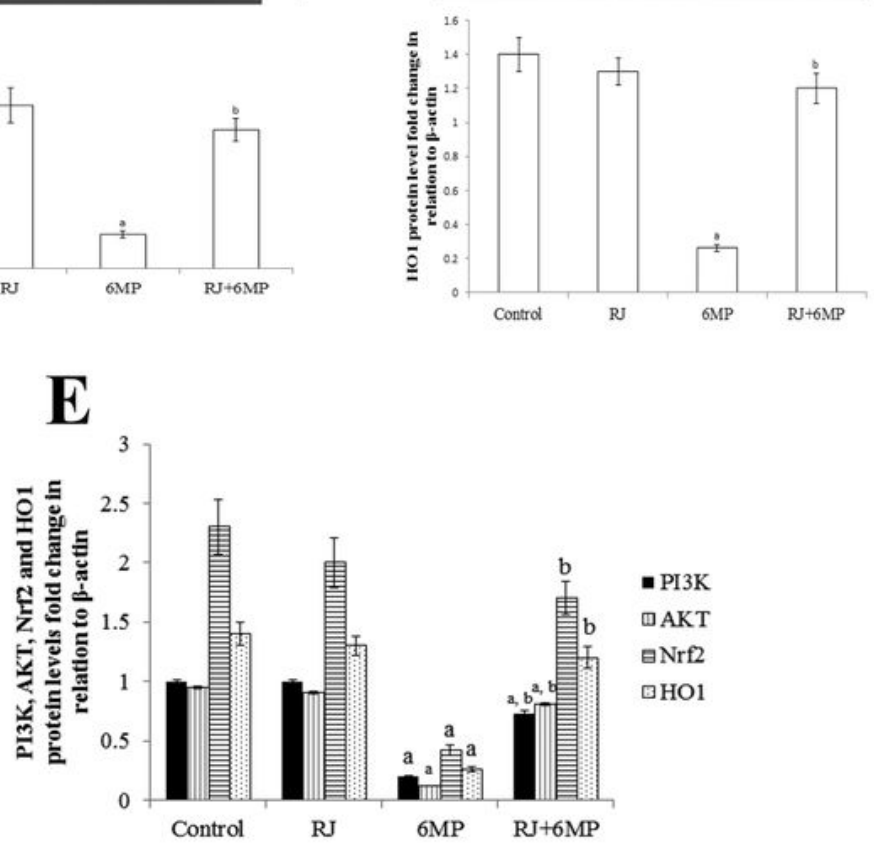

Figure 4 
Effect of 6MP on testicular PI3K/AKT-Nrf2/H01 pathway-related protein expression. A) The most representative images of western blotting and the expression level of PI3K protein relative to $\beta$-actin. B) The most representative images of western blotting and the expression level of AKT protein relative to $\beta$ actin. C) The most representative images of western blotting and the expression level of Nrf2 protein relative to $\beta$-actin. D) The most representative images of western blotting and the expression level of $\mathrm{HO} 1$ protein relative to $\beta$-actin. E) comparison of $\mathrm{PI} 3 \mathrm{~K}, \mathrm{AKT}, \mathrm{Nrf2}, \mathrm{HO} 1$ protein levels in different treated groups. Values are means $\pm S E, n=10$. a indicates a significant difference compared to control group at $P<0.05$. $b$ indicates a significant difference compared to 6MP group at $P<0.05$. 


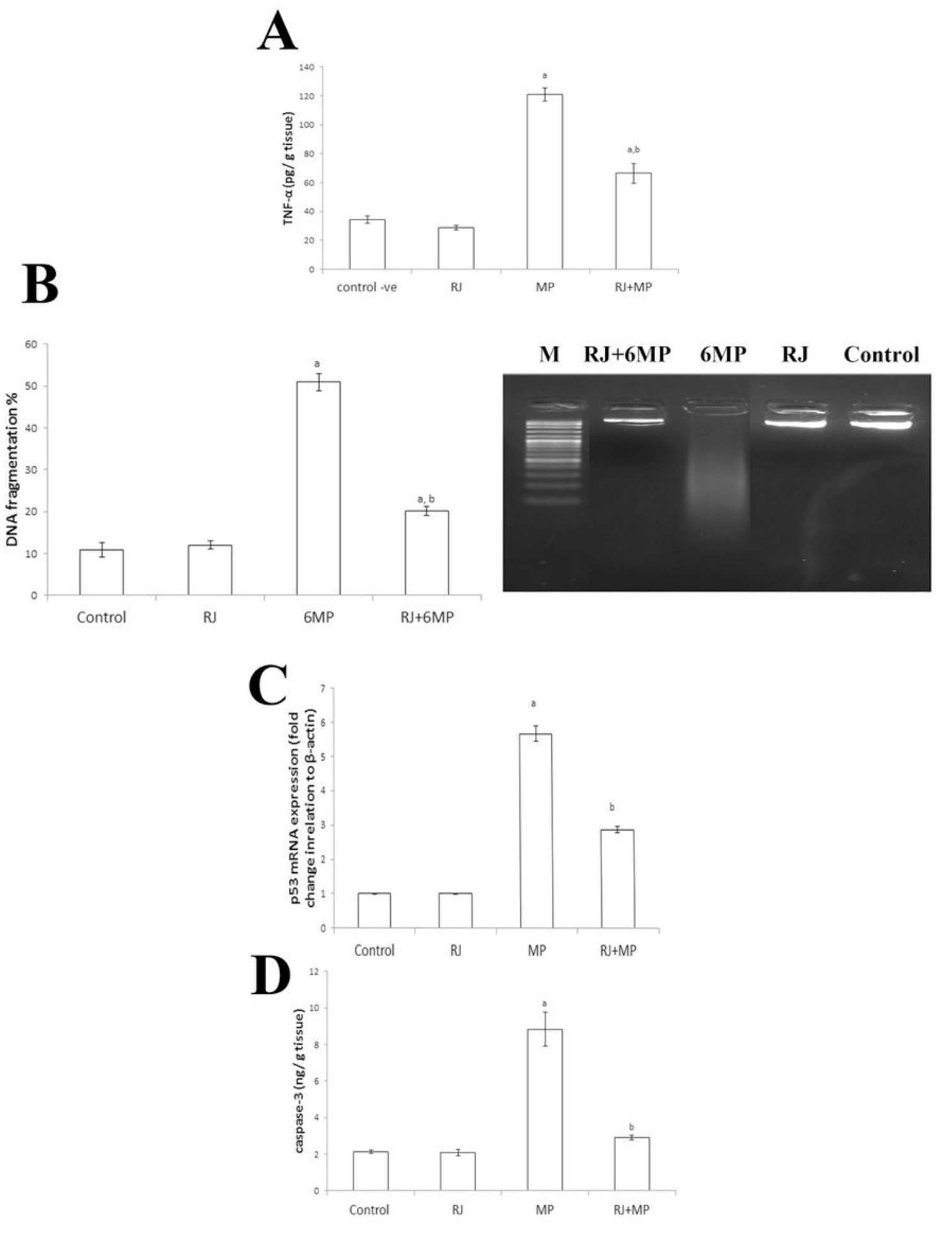

Figure 5

The effect of 6MP on testicular TNF-a, DNA fragmentation \%, P53 mRNA and caspase-3. A) TNF-a; tumor necrosis factor alpha in different treated groups. B) DNA fragmentation \% in different treated groups. C) P53 mRNA expression by real time PCR in different treated groups. E) Caspase-3 level in different treated groups. Values are means $\pm S E, n=10$. a indicates a significant difference compared to control group at $P<0.05$. $b$ indicates a significant difference compared to 6MP at $P<0.05$. 
Page 24/24 